\title{
ON LAMB'S PLANE PROBLEM IN MICROPOLAR VISCOELASTIC HALF-SPACE WITH STRETCH
}

\author{
RAJNEESH KUMAR \\ Department of Mathematics, \\ Doaba College \\ Jalandhar, India \\ M.L. GOGNA \\ Department of Mathematics \\ Kurukshetra University \\ Kurukshetra, India \\ LOKENATH DEBNATH \\ Department of Mathematics \\ University of Central Florida \\ Orlando, Florida 32816, U.S.A.
}

(Received November 1, 1988 and in revised form December 30, 1988)

\begin{abstract}
A study is made of Lamb's plane problem in micropolar viscoelastic halfspace with stretch. The viscoelasticity is characterized by the rate-dependent theories of micro-viscoelasticity generalizing the classical Kelvin-Voigt theory. The displacement components, force stress, couple stress and vector first moment are obtained for a half-space subjected to an arbitrary normal load. Two particular cases of a horizontal force and a torque which are time harmonic have been considered. Several limiting cases are obtained as special cases of the present analysis.
\end{abstract}

KEYWORDS AND PHRASES. Lamb's problem, Micropolar Viscoelastic Medium and Fourier Trans forms.

1980 AMS SUBJECT CLASSIFICATION CODE. 73D30.

1. INTRODUCTION.

Lamb's problem [1] has received considerable attention by several researchers in different elastic media with various kinds of loading. In particular, Nowacki and Nowacki [2] have studied the Lamb problem in micropolar elastic media. Chadha [3] has investigated the Lamb problem in micropolar elastic media and discussed the propagation of waves in a semi-infinite micropolar elastic solid due to loading at the plane boundary of a semi-half space. Acharya and Sengupta [4] have recently studied the same problem in a thermo-elastic medium under the influence of temperture. They have examined the longitudinal and transverse thermo-elastic waves in a micropolar 
semi-infinite space bounded by a plane in which a normal loading is applied. In a recent paper by Ray and Sengupta [5], a study is made of a two-dimensional waves in a micropolar thermo-viscoelastic medium. More recently, Kumar and Chadha [6] and Chadha, Kumar and Debnath [7] have made an investigation of the Lamb problem in a microelastic half-space with stretch, and in a thermoelastic micropolar medium with stretch.

Based upon the linear theory of micropolar viscoelastic waves due to Eringen [89], a study is made of Lamb's plane problem in micropolar viscoelastic half-space with the effect of stretch. The displacement components, force stress, couple stress, and vector first moment are determined for a half-space subjected to an arbitrary normal load. Two particular cases of a horizontal force and a torque which are time harmonic are cited as examples of the general theory.

\section{BASIC CONSTITUTIVE AND FIELd EQUATIONS.}

Making reference to Eringen [8-10] and Nowacki [11], the constitutive and field equations for micropolar viscoelastic solids with stretch in the absence of body forces, body moments and stretch forces are

$$
\begin{aligned}
& t_{j 1}=\left(\lambda+\lambda_{1} \frac{\partial}{\partial t}\right) u_{k, k} \delta_{j i}+\left\{(\mu-\alpha)+\left(\mu_{1}-\alpha_{1}\right) \frac{\partial}{\partial t}\right\}\left(u_{1, j}+u_{j, i}\right) \\
& +2\left(\alpha+\alpha_{1} \frac{\partial}{\partial t}\right)\left(u_{i, j}-\varepsilon_{k j i} \omega_{k}\right) \text {, } \\
& \left(m_{j i}=\beta_{0} \varepsilon_{k j i} \Phi, k+\left(\beta+\beta_{1} \frac{\partial}{\partial t}\right) w_{k, k} \delta_{j i}+\left\{(\gamma-\varepsilon)+(\gamma-\varepsilon) \frac{\partial}{\partial t}\right\} \omega_{j, i}\right. \\
& +\left[(\gamma+\varepsilon)+(\gamma+\varepsilon) \frac{\partial}{\partial t}\right] \omega_{j, 1}, \\
& \lambda_{j}=\alpha_{0} \Phi_{, j}+\frac{1}{3} \beta_{0} \varepsilon_{k j i} u_{k, i} \text {, } \\
& {\left[(\mu+\alpha)+\left(\mu_{1}+\alpha_{1}\right) \frac{\partial}{\partial t}\right] \nabla^{2} \underline{u}+\left[(\lambda+\mu-\alpha)+\left(\lambda_{1}+\mu_{1}-\alpha_{1}\right) \frac{\partial}{\partial t}\right] \operatorname{grad} \operatorname{div} \underline{u}} \\
& +2\left(\alpha+\alpha_{1} \frac{\partial}{\partial t}\right) \operatorname{rot} \underline{\omega}=\rho \frac{\partial^{2} \underline{u}}{\partial t^{2}} \text {, } \\
& {\left[(\gamma+\varepsilon)+\left(\gamma_{1}+\varepsilon_{1}\right) \frac{\partial}{\partial t}\right] \nabla^{2} \underline{\omega}+\left[(\gamma+\beta-\varepsilon)+\left(\gamma_{1}+\beta_{1}-\varepsilon\right) \frac{\partial}{\partial t}\right] \operatorname{grad} \operatorname{div} \underline{\omega}} \\
& -4\left(\alpha+\alpha_{1} \frac{\partial}{\partial t}\right) \underline{\omega}+2\left(\alpha+\alpha_{1} \frac{\partial}{\partial t}\right) \operatorname{rot} \underline{u}=\mathrm{J} \frac{\partial^{2} \underline{\omega}}{\partial t^{2}} \\
& \alpha_{0} \nabla_{\Phi}-\eta \Phi_{0}=\frac{J}{2} \frac{\partial \Phi^{2}}{\partial t^{2}}
\end{aligned}
$$

where $t_{j 1}, m_{j 1}$ and $\lambda_{j}$ are the components of force stress, couple stress and vector first moment, $\rho$ is the density, $J$ is the rotational inertia, $\underline{u}$ is the displacement vector, $\underline{\omega}$ is the microrotation vector and $\Phi$ is the scalar microstretch, and $\lambda, \mu, \alpha, \beta, \gamma, \varepsilon, \alpha_{0}, \beta_{0}, \eta_{0}$ are material constants. 


\section{BASIC EQUATIONS AND THE BOUNDARY CONDITIONS}

We consider a semi-infinite homogeneous, isotropic, micropolar viscoelastic solid with stretch. We take the rectangular Cartesian coordinates $x, y$, and $z$ with the origin at the plane boundary of the half-space, $z>0$ and the $z$-axis is normal to the medium. We assume that there is a uniform stretch in the $x$-direction and a given loading function $g(x, t)$ normal to the free surface $z=0$.

We consider the two-dimensional problem so that the displacement and microrotation are independent of the $y$ coordinate. Hence, we may write $\underline{u}=\left(u_{1}, 0, u_{3}\right)$ and $\underline{\omega}=\left(0, w_{2}, 0\right)$. We introduce displacement potentials $\phi(x, z, t)$ and $\psi(x, z, t)$ defined by $u_{1}=\phi_{x}+\psi_{z}$ and $u_{3}=\phi_{z}-\psi_{x} \cdot$

Consequently, equations $(2.4)-(2.6)$ can be written in terms of $\phi$ and $\psi$ as

$$
\begin{aligned}
& {\left[\left(c_{1}^{2}+c_{1}^{\prime 2} \frac{\partial}{\partial t}\right) \nabla^{2}-\frac{\partial^{2}}{\partial t^{2}}\right] \phi=0,} \\
& {\left[\left(c_{2}^{2}+c_{2}^{\prime 2}\right) \frac{\partial}{\partial t} \nabla^{2}-\frac{\partial^{2}}{\partial t^{2}}\right] \psi-\left(p_{1}+p_{1}^{\prime} \frac{\partial}{\partial t}\right) w_{2}=0,} \\
& {\left[\left(c_{3}^{2}+c_{3}^{\prime 2} \frac{\partial}{\partial t}\right) \nabla^{2}-\left(\gamma_{2}+\gamma_{2}^{\prime} \frac{\partial}{\partial t}+\frac{\partial^{2}}{\partial t^{2}}\right)\right] \omega_{2}+\frac{1}{2}\left(\gamma_{2}+\gamma_{2}^{\prime} \frac{\partial}{\partial t}\right) \nabla^{2} \psi=0,} \\
& {\left[\nabla^{2}-\gamma_{3}-\frac{1}{c_{4}^{2}} \frac{\partial^{2}}{\partial t^{2}}\right] \Phi=0,}
\end{aligned}
$$

where

$$
\begin{aligned}
& \nabla^{2} \equiv \frac{\partial^{2}}{\partial x^{2}}+\frac{\partial^{2}}{\partial z^{2}}, c_{1}^{2}=(\lambda+2 \mu) / \rho, c_{1}^{\prime 2}=\left(\lambda_{1}+2 \mu_{1}\right) / \rho, c_{2}^{2}=(\mu+\alpha) / \rho \\
& c_{2}^{\prime 2}=\left(\mu_{1}+\alpha_{1}\right) / \rho, c_{3}^{2}=(\gamma+\varepsilon) / J, c_{3}^{\prime 2}=\left(\gamma_{1}+\varepsilon_{1}\right) / J, \\
& c_{4}^{2}=2 \alpha_{0} / J, p_{1}=2 \alpha / \rho, p_{1}^{\prime}=2 \alpha_{1} / \rho, \gamma_{2}=4 \alpha / J, \gamma_{2}^{\prime}=4 \alpha_{1} / J, \gamma_{3}=\eta_{0} / \alpha_{0} .
\end{aligned}
$$

Eliminating $\psi$ or $\omega_{2}$ from (3.2) and (3.3), we obtain

$$
\begin{aligned}
{\left[\left\{\left(c_{2}^{2}+c_{2}^{\prime 2} \frac{\partial}{\partial t}\right) \nabla^{2}\right.\right.} & \left.-\frac{\partial^{2}}{\partial t^{2}}\right\}\left\{\left(c_{3}^{2}+c_{3}^{\prime 2} \frac{\partial}{\partial t}\right) \nabla^{2}-\left(\gamma_{2}+\gamma_{2}^{\prime} \frac{\partial}{\partial t}+\frac{\partial^{2}}{\partial t^{2}}\right)\right\} \\
& \left.+\frac{1}{2}\left(p_{1}+p_{1}^{\prime} \frac{\partial}{\partial t}\right)\left(\gamma_{2}+\gamma_{2}^{\prime} \frac{\partial}{\partial t}\right) \nabla^{2}\right]\left(\psi, \omega_{2}\right)=0
\end{aligned}
$$

The boundary conditions of the problem are

where

$$
t_{33}=-g(x, t), t_{31}=m_{32}=\lambda_{3}=0 \quad \text { at } z=0
$$

$$
\begin{aligned}
& t_{33}=\left(\lambda+\lambda_{1} \frac{\partial}{\partial t}\right) \nabla^{2} \phi+2\left(\mu+\mu_{1} \frac{\partial}{\partial t}\right)\left(\frac{\partial^{2} \phi}{\partial z^{2}}-\frac{\partial^{2} \psi}{\partial x \partial z}\right), \\
& t_{31}=\left(\mu+\mu_{1} \frac{\partial}{\partial t}\right)\left(2 \frac{\partial^{2} \phi}{\partial z \partial z}+\frac{\partial^{2}}{\partial z^{2}}-\frac{\partial^{2}}{\partial x^{2}}\right)+\left(\alpha+\alpha_{1} \frac{\partial}{\partial t}\right)\left(\nabla^{2} \psi-2 \omega_{2}\right),
\end{aligned}
$$




$$
\begin{aligned}
& \mathrm{m}_{32}=\left[(\gamma+\varepsilon)+\left(\gamma_{1}+\varepsilon_{1}\right) \frac{\partial}{\partial t}\right] \frac{\partial \omega_{2}}{\partial z}-\beta_{0} \frac{\partial \Phi}{\partial x} \\
& \lambda_{3}=\alpha_{0} \frac{\partial \Phi}{\partial z}+\frac{\beta_{0}}{3} \frac{\partial \omega_{2}}{\partial x}
\end{aligned}
$$

In addition, we assume that $\phi, \psi, \omega_{2}$ and $\phi$ tend to zero as $z \rightarrow \infty$.

4. SOLUTION OF THE PROBLEM.

We apply the double Fourier transform $\tilde{\tilde{f}}(k, z, s)$ of $f(x, z, t)$ defined by (Myint-U and Debnath, [13]) as

$$
\tilde{\tilde{f}}(k, z, s)=\frac{1}{2 \pi} \int_{-\infty}^{\infty} \int \exp \{1(k x+s t)\} f(x, z, t) d x d t
$$

and its inverse is given by

$$
\begin{aligned}
& f(x, z, t)=\frac{1}{2 \pi} \iint_{-\infty}^{\infty} \exp [-i(k x+s t)] \tilde{\tilde{f}}(k, z, s) d k d s \\
& \text { Applications of this transforms to equations }(3.1)-(3.4) \text { gives } \\
& {\left[\frac{d^{2}}{d z^{2}}-s_{1}^{2}\right] \tilde{\bar{\phi}}=0,} \\
& {\left[\left(\frac{d^{2}}{d z^{2}}-s_{2}^{2}\right)\left(\frac{d^{2}}{d z^{2}}-s_{3}^{2}\right)\right]\left(\frac{\left.\tilde{\psi}, \tilde{\omega}_{2}\right)=0,}{\left(\frac{d^{2}}{d z^{2}}-s_{4}^{2}\right) \tilde{\bar{\phi}}=0,}\right.}
\end{aligned}
$$

where

$$
\begin{aligned}
& s_{1}^{2}=k^{2}-\frac{s^{2}}{c_{1}^{2}-1 s c_{1}^{2}}, \\
& s_{2}^{2}+s_{3}^{2}-2 k^{2}=-\frac{s^{2}}{c_{2}^{2}-i s c_{2}^{\prime 2}}+\frac{r_{2}-1 s \gamma_{2}^{\prime-s} s^{2}}{c_{3}^{2}-1 s c_{3}^{\prime 2}}-\frac{1}{2} \frac{\left(p_{1}-i s p_{1}^{\prime}\right)\left(\gamma_{2}-1 s \gamma_{2}^{\prime}\right)}{\left(c_{2}^{2}-1 s c_{2}^{\prime 2}\right)\left(c_{3}^{2}-1 s c_{3}^{\prime 2}\right)} \\
& \left(s_{2}^{2}-k^{2}\right)\left(s_{3}^{2}-k^{2}\right)=\frac{s^{2}\left[s^{2}-\left(\gamma_{2}-1 s \gamma_{2}^{\prime}\right)\right]}{\left(c_{2}^{2}-1 s c_{2}^{\prime 2}\right)\left(c_{3}^{2}-1 s c_{3}^{\prime 2}\right)} \\
& s_{4}^{2}=k^{2}+\gamma_{3}-\frac{s^{2}}{c_{4}^{2}}
\end{aligned}
$$

The solutions of $(4.2)-(4.4)$ with the boundary conditions at infinity are

$$
\begin{aligned}
& \tilde{\bar{\phi}}=A e^{-s} 1^{z}, \tilde{\psi}=B e^{-s} 2^{z}+C e^{-s} 3^{z} \\
& \tilde{\omega}_{2}=B_{1} e^{-s} 2^{z}+C_{1} e^{-s} 3^{z}, \tilde{\Phi}=D e^{-s / z}
\end{aligned}
$$


where $i t$ is assumed that $\operatorname{Re}\left(s_{j}\right)>0$ for $f=1,2,3,4$ and

$$
\begin{aligned}
& B_{1}=a_{2} B, C_{1}=a_{3} C, \\
& a_{j}=\frac{1}{p_{1}-18 p_{1}^{i}}\left[\left(c_{2}^{2}-1 s c_{2}^{2}\right)\left(s_{j}^{2}-k^{2}\right)+s^{2}\right], j=2,3 \\
& \quad \text { Applying the Fourier transforms (3.1) to the boundary conditions (3.6abcd) - } \\
& (2.10) \text { and combined with }(4.9 a b)-(4.10 a b) \text {, we obtain } \\
& (A, B, C, D)=\left\{\frac{\Delta_{1}}{\Delta}, \frac{\Delta_{2}}{\Delta}, \frac{\Delta_{3}}{\Delta}, \frac{\Delta_{4}}{\Delta}\right\} \tilde{g}(k, s)
\end{aligned}
$$

where

$$
\begin{aligned}
& \Delta=\left(r_{3} t_{4}-r_{4} t_{3}\right)\left(q_{1} m_{2}-q_{2} m_{1}\right)+\left(r_{2} t_{4}-r_{4} t_{2}\right)\left(q_{3} m_{1}-q_{1} m_{3}\right), \\
& \Delta_{1}=m_{3}\left(r_{2} t_{4}-r_{4} t_{2}\right)-m_{2}\left(r_{3} t_{4}-r_{4} t_{3}\right), \\
& \Delta_{2}=m_{1}\left(r_{3} t_{4}-r_{4} t_{3}\right), \Delta_{3}=m_{1}\left(r_{4} t_{2}-r_{2} t_{4}\right), \\
& \Delta_{4}=m_{1}\left(r_{2} t_{3}-r_{3} t_{2}\right) \text {. } \\
& q_{j}=-21 \mu * k s_{j}, \\
& j=1 \\
& 21 \mu * \mathrm{ks}_{\mathrm{j}}, \\
& j=2,3 \\
& j=1 \\
& m_{j}= \\
& \mu^{*}\left(k^{2}+s_{j}^{2}\right)+\alpha *\left(s_{j}^{2}-k^{2}-2 a_{j}\right) \quad j=2,3 \\
& \left(\gamma^{*}+\varepsilon^{*}\right) 8_{j} a_{j}, \quad j=2,3 \\
& \mathbf{r}_{j}= \\
& \text { i } \beta_{0} k \text {, } \\
& j=4 \\
& 1 \beta_{o} k_{j}, \quad j=2,3 \\
& t_{j}= \\
& 3 \alpha_{0}{ }^{\mathrm{j}} \\
& j=4 \\
& \lambda^{*}=\lambda-18 \lambda_{1}, \mu^{*}=\mu-18 \mu_{1}, \alpha^{*}=\alpha-18 \alpha, \gamma_{1}^{*}=\gamma-18 \gamma_{1}, \varepsilon^{*}=\varepsilon-18 \varepsilon,
\end{aligned}
$$

Application of the inverse Fourier transformation to (4.9ab) - (4.10ab) gives

$$
\begin{aligned}
& \phi=\frac{1}{2 \pi} \int_{-\infty}^{\infty} \int_{-\infty}^{\infty} A e^{-s} 1^{z-1(k x+s t)} d k d s, \\
& \psi=\frac{1}{2 \pi} \int_{-\infty}^{\infty} \int_{-\infty}^{\infty}\left(B e^{-s} 2^{z}+C e^{-s} 3^{z}\right) e^{-i(k x+s t)} d k d s
\end{aligned}
$$




$$
\begin{aligned}
& \omega_{2}=\frac{1}{2 \pi} \int_{-\infty}^{\infty} \int_{-\infty}^{\infty}\left(\mathrm{a}_{2} \mathrm{Be}^{-s} 2^{z}+\mathrm{a}_{3} \mathrm{Ce}^{-s} 3^{z}\right) \mathrm{e}^{-1(k x+s t)} \mathrm{dkds} \\
& \Phi=\frac{1}{2 \pi} \int_{-\infty}^{\infty} \int_{-\infty}^{\infty} \mathrm{D} \mathrm{e}^{-s 4^{z-1(k x+s t)}} \mathrm{dkds} .
\end{aligned}
$$

Using (4.19) - (4.22), we obtain the formal solutions for the displacement components, microrotations, force stress, couple stress and vector first moment in the form

$$
\begin{aligned}
& u_{1}=-\frac{1}{2 \pi} \iint_{-\infty}^{\infty} F_{1}(z, k, s) \tilde{\bar{g}}(k, s) e^{-i(k x+s t)} d k d s, \\
& u_{3}=-\frac{1}{2 \pi} \iint_{-\infty}^{\infty} F_{2}(z, k, s) \tilde{g}(k, s) e^{-i(k x+s t)} d k d s, \\
& w_{2}=\frac{1}{2 \pi} \iint_{-\infty}^{\infty} F_{3}(z, k, s) \tilde{\tilde{g}}(k, s) e^{-1(k x+s t)} d k d s \\
& t_{33}=\frac{1}{2 \pi} \int_{-\infty}^{\infty} F_{4}(z, k, s) \tilde{\tilde{g}}(k, s) e^{-i(k s+s t)} d k d s, \\
& t_{31}=\frac{1}{2 \pi} \iint_{-\infty}^{\infty} F_{5}(z, k, s) \tilde{\bar{g}}(k, s) e^{-i(k s+s t)} d k d s, \\
& m_{32}=-\frac{1}{2 \pi} \iint_{-\infty}^{\infty} F_{6}(z, k, s) \tilde{g}(k, s) e^{-i(k x+s t)} d k d s, \\
& \lambda_{3}=-\frac{1}{6 \pi} \iint_{-\infty}^{\infty} F_{7}(z, k, s) \tilde{\tilde{g}}(k, s) e^{-i(k x+s t)} d k d s,
\end{aligned}
$$

where

$$
\begin{aligned}
& F_{1}(z, k, 8)=\frac{1}{\Delta}\left[1 k \Delta_{1} e^{-8} 1^{z}+s_{2} \Delta_{2} e^{-s} 2^{z}+s_{3} \Delta_{3} e^{-s} 3^{z}\right], \\
& F_{2}(z, k, s)=\frac{1}{\Delta}\left[s_{1} \Delta_{1} e^{-s} 1^{z}-i k\left(\Delta_{2} e^{-s} 2^{z}+\Delta_{3} e^{-s} 3^{z}\right)\right] \\
& F_{3}(z, k, 8)=\frac{1}{\Delta}\left(a_{2} \Delta_{2} e^{-8} 2^{z}+a_{3} \Delta_{3} e^{-8} 3^{z}\right) \text {, } \\
& F_{4}(z, k, s)=\frac{1}{\Delta}\left[\left\{(\lambda *+2 \mu *) s_{1}^{2}-\lambda * k^{2}\right\} \Delta_{1} e^{-8} 1^{z}-2 i \mu k k\left(s_{2} \Delta_{2} e^{-s} 2^{z}+s_{3} \Delta_{3} e^{-8} 3^{z}\right)\right] \\
& F_{5}(z, k, s)=\frac{1}{\Delta}\left[2 i \mu^{* k} s_{1} \Delta_{1} e^{-s} 1^{z}+\left\{\left(\mu^{*}+\alpha^{*}\right) s_{2}^{2}+\left(\mu^{*}-\alpha^{*}\right) k^{2}-2 \alpha^{*} \alpha_{2}\right\} \Delta_{2} e^{-s} 2^{z}\right. \\
& \left.+\left\{\left(\mu^{*}+\alpha^{*}\right) s_{3}^{2}+\left(\mu^{*}-\alpha^{*}\right) k^{2}-2 \alpha^{*} a_{3}\right\} \Delta_{3} e^{-8} 3^{2}\right\}, \\
& F_{6}(z, k, 8)=\frac{1}{\Delta}\left[\left(\gamma^{*}+\varepsilon^{*}\right)\left(a_{2} s_{2} \Delta_{2} e^{-s} 2^{z}+a_{3} s_{3} \Delta_{3} e^{-8} 3^{z}\right)-1 \beta_{0} k \Delta_{4} e^{-8} 4^{z}\right], \\
& F_{7}(z, k, s)=\frac{1}{\Delta}\left[1 \beta_{0} k\left(a_{2} \Delta_{2} e^{-s} 2^{z}+a_{3} \Delta_{3} e^{-s} 3^{z}\right)+\alpha_{0} s_{4} \Delta_{4} e^{-s} 4^{z}\right]
\end{aligned}
$$


5. PARTICULAR SOLUTIONS OF INTEREST.

We first consider a time harmonic concentrated normal load in the form $g(x, t)=P \delta(x) e^{i \omega t}$

where $P$ is a constant and $\delta(x)$ is the Dirac delta function and $\omega$ is the frequency. The double Fourier transform of $g(x, t)$ is

$$
\tilde{\bar{g}}(k, s)=P \delta(s-\omega)
$$

Consequently the solutions assume the form

$u_{1}=-\frac{P}{2 \pi} \int_{-\infty}^{\infty}\left[F_{1}(z, k, s)\right]_{s=\omega} \exp [1(k x+\omega t)] d k$

$u_{3}=-\frac{P}{2 \pi} \int_{-\infty}^{\infty}\left[F_{2}(z, k, 8)\right]_{s=\omega} \exp [-1(k x+\omega t)] d k$

$\omega_{2}=\frac{P}{2 \pi} \int_{-\infty}^{\infty}\left[F_{2}(z, k, s)\right]_{8=\omega} \exp [-1(k x+\omega t)] d k$

$t_{33}=\frac{P}{2 \pi} \int_{-\infty}^{\infty}\left[F_{4}(z, k, s)\right]_{s=\omega} \exp [-1(k x+\omega t)] d k$

$t_{31}=\frac{P}{2 \pi} \int_{-\infty}^{\infty}\left[F_{5}(z, k, s)\right]_{s=\omega} \exp [-1(k x+\omega t)] d k$

$m_{32}:-\frac{P}{2 \pi} \int_{-\infty}^{\infty}\left[F_{6}(z, k, s)\right]_{s=\omega} \exp [-1(k x+\omega t)] d k$

$\lambda_{3}=-\frac{P}{6 \pi} \int_{-\infty}^{\infty}\left[F_{7}(z, k, s)\right]_{s=\omega} \exp [-1(k x+\omega t)] d k$

Neglecting the viscous effects, we obtain the corresponding results for the micropolar elastic half space with stretch

$$
\begin{aligned}
& u_{1}=-P \int_{-\infty}^{\infty}\left[G_{1}(z, k, s)\right]_{s=\omega} \exp [-1(k x+\omega t)] d k \\
& u_{3}=-\frac{P}{2 \pi} \int_{-\infty}^{\infty}\left[G_{2}(z, k, s)\right]_{s=\omega} \exp [-1(k x+\omega t)] d k \\
& w_{2}=\frac{P}{2 \pi} \int_{-\infty}^{\infty}\left[G_{3}(z, k, s)\right]_{s=\omega} \exp [-1(k x+\omega t)] d k \\
& t_{33}=\frac{P}{2 \pi} \int_{-\infty}^{\infty}\left[G_{4}(z, k, s)\right]_{s=\omega} \exp [-1(k x+\omega t)] d k \\
& t_{31}=\frac{P}{2 \pi} \int_{-\infty}^{\infty}\left[G_{5}(z, k, s)\right]_{s=\omega} \exp [-1(k x+\omega t)] d k \\
& m_{32}=-\frac{P}{2 \pi} \int_{-\infty}^{\infty}\left[G_{6}(z, k, s)\right]_{s=\omega} \exp [-1(k x+\omega t)] d k \\
& \lambda_{3}=-\frac{P}{2 \pi} \int_{-\infty}^{\infty}\left[G_{7}(z, k, s)\right]_{s=\omega} \exp [-i(k x+\omega t)] d k
\end{aligned}
$$




\section{where}

$$
\begin{aligned}
& G_{1}(z, k, s)=\frac{1}{\Delta_{0}}\left[1 k \Delta_{11} e^{-s} 11^{z}+s_{21} \Delta_{21} e^{-s} 21^{z}+s_{31} \Delta_{31} e^{-s} 31^{z}\right], \\
& G_{2}(z, k ; s)=\frac{1}{\Delta_{0}}\left[s_{11} \Delta_{11} e^{-s} 11^{z}-i k\left(\Delta_{21} e^{-s} 21^{z}+\Delta_{31} e^{-s} 31^{z}\right)\right] \text {, } \\
& G_{3}(z, k, s)=\frac{1}{\Delta_{0}}\left(a_{21} \Delta_{21} e^{-s} 21^{z}+a_{31} \Delta_{31} e^{-s} 31^{z}\right), \\
& G_{4}(z, k, s)=\frac{1}{\Delta_{0}}\left[\left\{(\lambda+2 \mu) s_{11}^{2}-\lambda k^{2}\right\} \Delta_{11} e^{-s} 11^{z}-21 \mu k\left(s_{21} \Delta_{21} e^{-s} 21^{z}\right.\right. \\
& \left.\left.+s_{31} \Delta_{31} e^{-s} 31^{2}\right)\right] \\
& G_{5}(z, k, s)=\frac{1}{\Delta_{0}}\left\{2 i \mu k s{ }_{11} \Delta_{11} e^{-s} 11^{z}+\left\{(\mu+\alpha) s_{21}^{2}+(\mu-\alpha) k^{2}-2 \alpha a_{21}\right\} \Delta_{21} e^{-8} 21^{z}\right. \\
& \left.+\left\{(\mu+\alpha) s_{31}^{2}+(\mu-\alpha) k^{2}-2 \alpha a_{31}\right\} \Delta_{31} e^{-8} 31^{z}\right\}, \\
& \left.G_{6}(z, k, s)=\frac{1}{\Delta_{0}}\left[(\gamma+\varepsilon)\left(a_{21} s_{21} \Delta_{21} e^{-s} 21^{z}+a_{31} s_{31} e^{-s} 31^{z}\right)-1 \beta_{0} k \Delta_{41} e^{-s} 4^{z}\right)\right], \\
& G_{7}(z, k, s)=\frac{1}{\Delta_{0}}\left[1 \beta_{0} k\left(a_{21} \Delta_{21} e^{-8} 21^{z}+a_{31} \Delta_{31} e^{-8} 31^{z}\right)+3 \alpha_{0} s \Delta_{4} 1^{-8} e^{z}\right] \text {, } \\
& s_{11}^{2}=k^{2}-\frac{s^{2}}{c_{1}^{2}} \text {, } \\
& s_{21}^{2}+s_{31}^{2}-2 k^{2}=-\left[s^{2}\left(\frac{1}{c_{2}^{2}}+\frac{1}{c_{3}^{2}}\right)-\frac{\gamma_{2}}{c_{3}^{2}}\left(1-\frac{p_{1}}{2 c_{2}^{2}}\right)\right] \text {, } \\
& \left.s_{21}^{2}-k^{2}\right)\left(s_{31}^{2}-k^{2}\right)=\frac{s^{2}\left(s^{2}-r_{2}\right)}{c_{2}^{2} c_{3}^{2}} \text {, } \\
& a_{j 1}=\frac{1}{p_{1}}\left(s_{j 1}^{2}+\frac{s^{2}}{c_{2}^{2}}-k^{2}\right), \quad j=2,3 \\
& \Delta_{0}=\left(r_{31} t_{4}-r_{4} t_{31}\right)\left(q_{11} m_{21}-q_{21} m_{11}\right)+\left(r_{21} t_{4}-r_{4} t_{21}\right)\left(q_{31} m_{1}-q_{11} m_{31}\right), \\
& \Delta_{11}=m_{31}\left(r_{21} t_{4}-r_{4} t_{21}\right)-m_{21}\left(r_{31} t_{4}-r_{4} t_{31}\right) \\
& \Delta_{21}=m_{11}\left(r_{31} t_{4}-r_{4} t_{31}\right), \Delta_{31}=m_{11}\left(r_{4}{ }_{21}-r_{21} t_{4}\right), \\
& \left.\left.\Delta_{41}=m_{11}\right) r_{21} t_{31}-r_{31} t_{21}\right) \text {, } \\
& q_{j 1}=\left\{\begin{array}{cc}
(\lambda+2 \mu) s_{j 1}^{2}-\lambda k^{2}, & j=1 \\
-21 \mu k s_{j 1} & j=2,3
\end{array}\right\}
\end{aligned}
$$




$$
\begin{array}{ll}
m_{j 1}={ }^{2 i \mu k s_{j l},} & j=1 \\
r_{j 1}=(\gamma+\alpha) s_{j 1}^{2}+(\mu-\alpha) k^{2}-2 \alpha a_{j 1}, & j=2,3 \\
t_{j 1}=1 B_{0} k_{j l} & j=2,3
\end{array}
$$

If we neglect both the viscous and stretch effects, we obtain the corresponding displacements, microrotation and stresses for the micorpolar elastic half space. For the sake of brevity, we avoid writing down these results.

We next consider the effects of the harmonic torque with its axis parallel to the z-axis in the form

$$
g(x, t)=M[\delta(x-a)-\delta(x+a)] e^{-i \omega t}
$$

where $M$ is the magnitude of the torque. The double Fourier transform of (5.32) gives $\tilde{\bar{g}}(k, s)=2 i M \sin k a \delta(s-\omega)$

Using this expression in the equations (4.23)-(4.29), we obtain

$$
\begin{aligned}
u_{1} & =-Q \int_{-\infty}^{\infty}\left[F_{1}(z, k, s)\right]_{s=\omega} \sin (k a) e^{-i k x} d k \\
u_{3} & -Q \int_{-\infty}^{\infty}\left[F_{2}(z, k, s)\right]_{s=\omega} \sin (k a) e^{-i k x} d k \\
\omega_{2} & =Q \int_{-\infty}^{\infty}\left[F_{3}(z, k, s)\right]_{s=\omega} \sin (k a) e^{-i k x} d k \\
t_{33} & =Q \int_{-\infty}^{\infty}\left[F_{4}(z, k, s)\right]_{s=\omega} \sin (k a) e^{-i k x_{d k}}, \\
t_{31} & =Q \int_{-\infty}^{\infty}\left[F_{5}(z, k, s)\right]_{s=\omega} \sin (k a) e^{-i k x_{d k}}, \\
m_{32} & =-Q \int_{-\infty}^{\infty}\left[F_{6}(z, k, s)\right]_{s=\bar{w}^{\prime \prime}} \sin (k a) e^{-i k x} d k, \\
\lambda_{3} & =-\frac{Q}{3} \int_{-\infty}^{\infty}\left(F_{7}(z, k, s)\right]_{s=\omega} \sin (k a) e^{-i k x} d k, \\
\text { where } & Q=\frac{i M}{\pi} e^{-i \omega t} .
\end{aligned}
$$

These results are in excellent agreement with those for the cases without viscous and/or stretch effects which have been discussed by several authors including McCarthy and Eringen [9], Kumar and Chadha [6] Nowacki and Nowaki [2], and Acharya and Sengupta [4]. 
ACKNOWLEDGEMENT.

This work is partially supported by the University of Central Florida.

\section{REFERENCES}

1. LAMB, H., On the Propagation of Tremors Over the Surface of Elastic Solid, Phil. Trans. Roy. Soc. London, A203 (1904), 1-42.

2. NOWACKI, W. and NOWACKI, W.K., The Plane Lamb's Problem in a Semi Infinite Micropolar Elastic Body, Arch. Mech. Stos. 21 (1969), 241-251.

3. CHADHA, T.K., Plane Lamb's Problem in a Semi-infinite Micropolar Elasticity, Gerlands Beitr. Geophysik. Leipzig, 88 (1979), 407-414.

4. ACHARYA, D. and Sengupta, P.R., Lamb's Plane Problem in Micropolar Elastic Medium under Influence of Temperature, Bull Polish Acad. Sci. Series Sci. Tech. 27(1979), 414-427.

5. ROY, S.K. and SENGUPTA, P.R., Two Dimensional Wave Propagation in a Micropolar Thermo Viscoelastic Medium, Bull. Acad. Polon. Sci. Ser. Sci. Techn. 34 (1986), 27-46.

6. KUMAR, R. and CHADHA, T.K., Lamb's Plane Problem in Micropolar Elastic Halfspace with Stretch, Bull. Acad. Polon. Sc1. Se1. Sc1. Techn 34(1986), 59-72.

7. CHADHA, T.K., KUMAR, R., and DEBNATH, L., Lamb's Plane Problem in a ThermoElastic Micropolar Medium with Stretch, Internat. J. Math. and Math. Sci. 10 (1987), 187-198.

8. ERINGIN, A.C., Linear Theory of Micropolar Visco-elasticity, Int. J. Eng. Sci. $\underline{5}$ (1967), 191-204.

9. McCARTHY, M.F. and ERINGIN, A.C., Micropolar Viscoelastic Waves, Int. J. Eng. Sci. 7 (1969), 447-458.

10. ERINGIN, A.C., Micropolar Elastic Solids with Stretch, Ari Kitabevi Math. $24(1971)$.

11. NOWACKI, W., Theory of Micropolar Elasticity, International Centre for Mechanical Sciences, Courses and Lectures No. 25, Springer Verlag, Berlin (1970). 


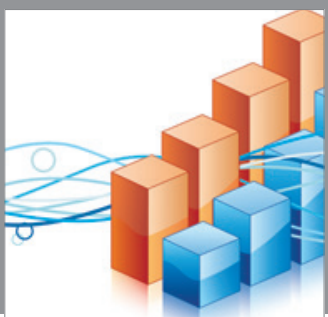

Advances in

Operations Research

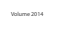

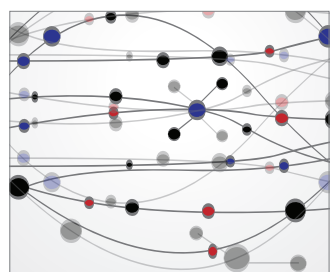

\section{The Scientific} World Journal
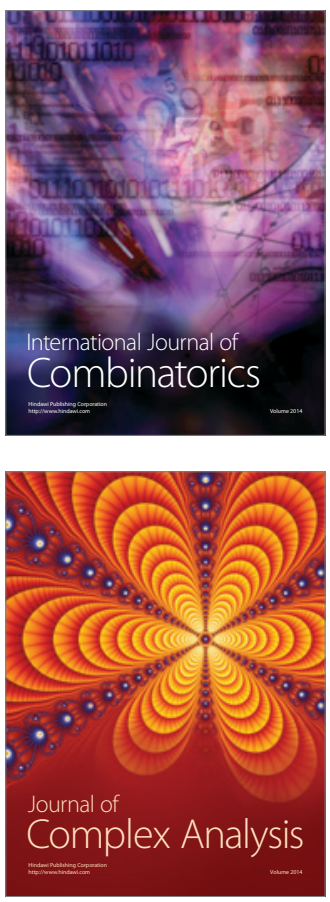

International Journal of

Mathematics and

Mathematical

Sciences
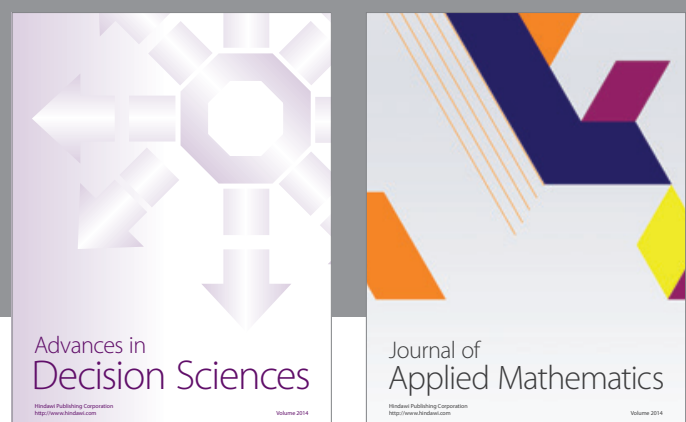

Journal of

Applied Mathematics
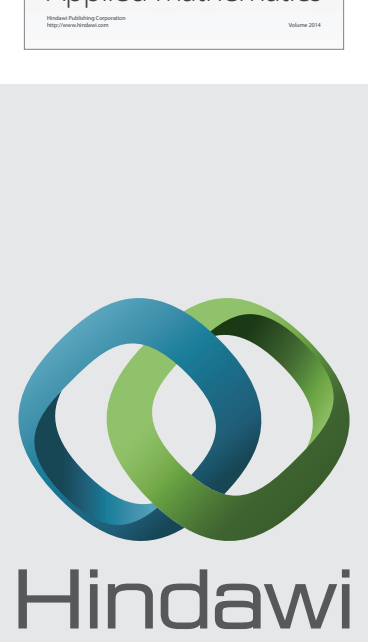

Submit your manuscripts at http://www.hindawi.com
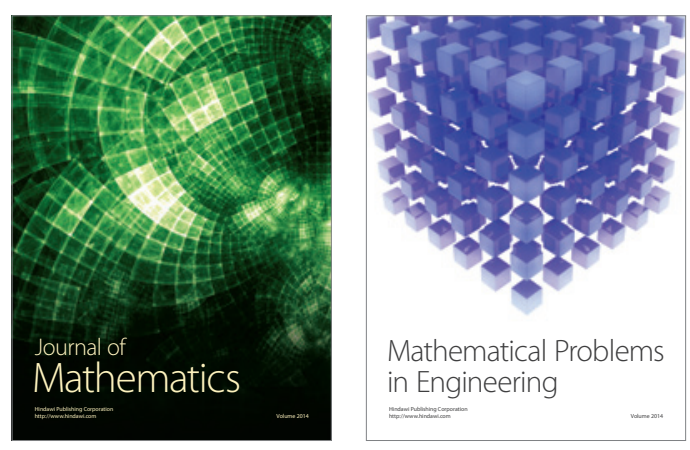

Mathematical Problems in Engineering
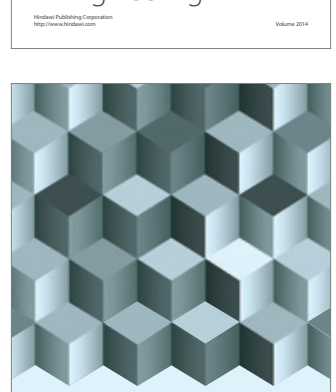

Journal of

Function Spaces
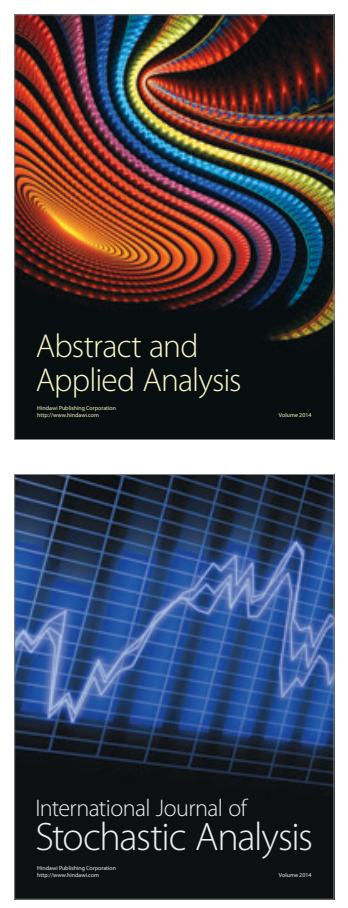

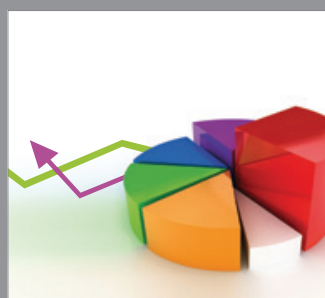

ournal of

Probability and Statistics

Promensencen
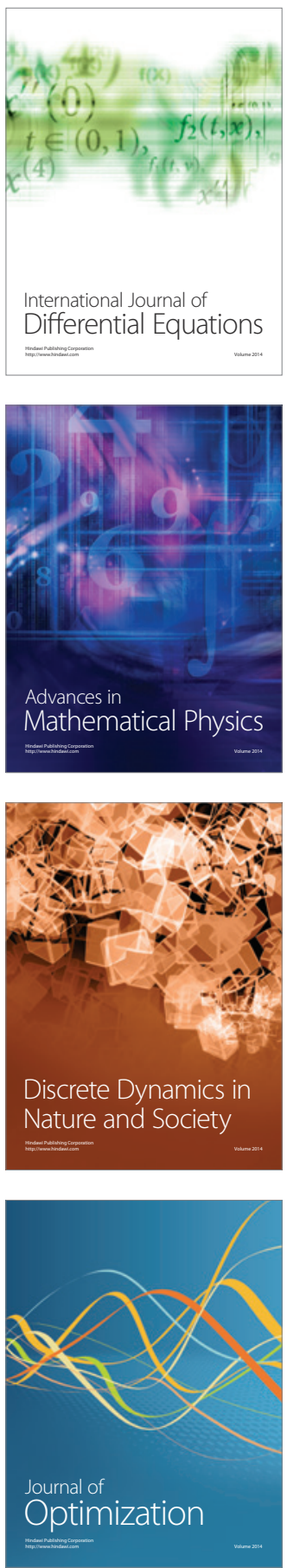